\title{
VELOCITY DISTRIBUTION AND PREDICTION OF FRICTION FACTOR AND PRESSURE GRADIENT IN A TURBULENT POROUS TUBE FLOW WITH INJECTION OR SUCTION
}

\author{
YUSHI HIRATA AND Ryuzo ITO \\ Department of Chemical Engineering, Osaka University, \\ Toyonaka 560
}

\begin{abstract}
An expression of velocity distribution of Stevenson's type is proposed for fully developed porous tube flow, on the basis of which the friction factor and the pressure gradient are analytically predicted. The prediction is in good agreement with the experimental results, but its applicability is restricted to a flow at $\widetilde{\boldsymbol{m}}_{w}<7.3 \times 10^{-3}$.
\end{abstract}

\section{Introduction}

Porous tube flow with injection or suction is regarded as a basic flow for studying heat and mass transfer in vaporization or condensation and mixing in a porous tube reactor.

There have been many investigations of the fluid dynamics of turbulent flow in the porous tube such as the axial pressure gradient, the friction factor and the velocity distribution. From the standpoint of estimating the effect of wall mass flux on the pressure gradient or the friction factor, the previous investigations may be divided into three groups; graphical presentation of the experimental results ${ }^{1,15)}$, experimental correlation with an injection or suction ratio $^{4,7,8)}$ and prediction using correlation of the velocity distribution ${ }^{9}$ or the mixing length ${ }^{5,6)}$. In the last group, the effect of injection or suction has been taken account of in the change of velocity distribution or mixing length. Prediction adopting the mixing length requires a numerical integration to solve the equation of motion.

Several authors ${ }^{10,12-14}$ presented expressions of velocity distribution in the boundary layer with injection or suction. $\mathrm{Na}^{9}$ applied Stevenson's expression $^{13)}$ to predicting the friction factor and the pressure gradient of porous tube flow, but it has not been sufficiently verified for porous tube flow because of the lack of velocity data measured in the wall region.

In this paper, an expression of velocity distribution of Stevenson's type is proposed for fully developed porous tube flow, on the basis of which the friction

Received January 5, 1981. Correspondence concerning this article should be addressed to $Y$. Hirata. factor and the pressure gradient are analytically predicted by extending Na's method to a wide range of Reynolds number.

The experimental results compared with the predictions are the same as those reported in the previous paper $^{43}$ which were obtained in a fully developed flow with injection or suction.

\section{Velocity Distribution}

Very near the wall, the equation of motion is simplified as

$$
-m_{w} \frac{\partial u}{\partial y}=-\frac{d p}{d x}+\frac{\partial \tau}{\partial y}
$$

where $m_{w}=\rho_{w} w_{w}$ is the radial mass velocity at the wall, which takes a positive value for suction and a negative value for injection in this study. Integration of Eq. (1) gives

$$
\tau^{+} \equiv \tau / \tau_{w}=1-m_{w}^{+} u^{+}+p^{+} y^{+}
$$

From Eq. (2), the velocity distribution in the viscous sublayer is derived as

$$
\begin{aligned}
u^{+}= & -\frac{\exp \left(-m_{w}^{+} y^{+}\right)-1}{m_{w}^{+}}+\frac{p^{+}}{m_{w}^{+2}}\left[\exp \left(-m_{w}^{+} y^{+}\right)\right. \\
& \left.-1+m_{w}^{+} y^{+}\right]
\end{aligned}
$$

If the mixing length $l=\kappa y$ is assumed to be applicable to the turbulent region in porous tube flow, one rewrites Eq. (2) as

$$
\frac{d u^{+}}{d y^{+}}=\frac{1}{\kappa y^{+}}\left[1-m_{w}^{+} u^{+}+p^{+} y^{+}\right]^{1 / 2}
$$

A solution of Eq. (4) can be obtained in a closed form only when $p^{+}=0$ or $m_{w}^{+}=0$. When $p^{+}=0$, the following solution is obtained. 


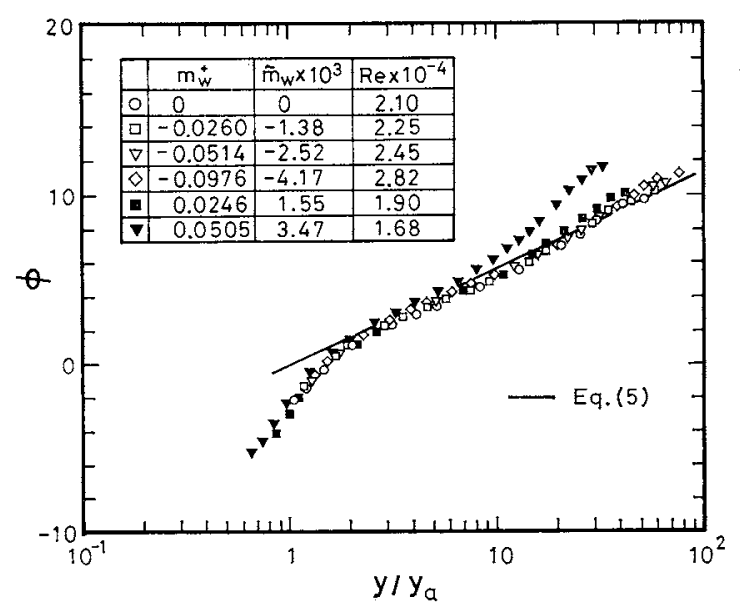

Fig. 1 Comparison of Eq. (5) with measured velocity distributions

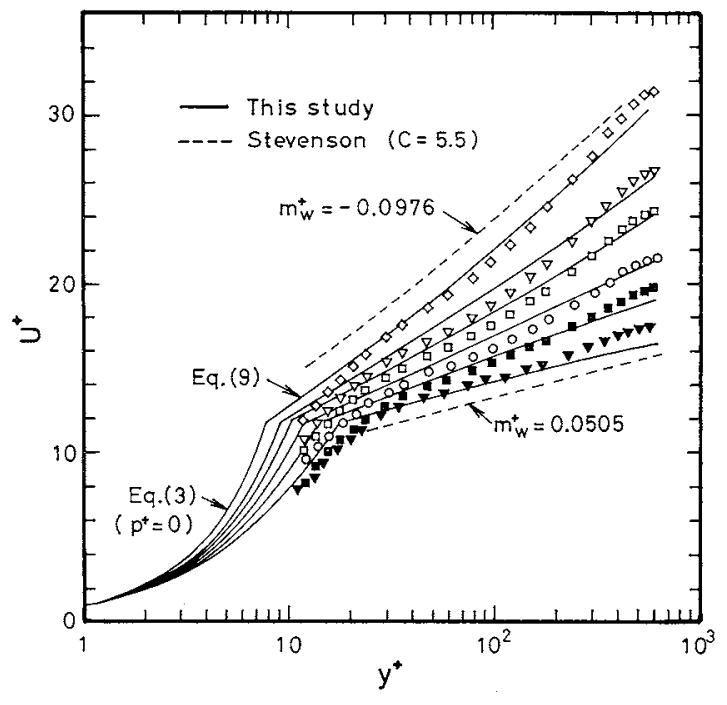

(Keys are the same as those in Fig. 1.)

Fig. 2 Comparison of Eq. (9) with measured velocity distributions

$$
\begin{aligned}
\phi & \equiv \frac{-2}{m_{w}^{+}}\left(\sqrt{1-m_{w}^{+} u^{+}}-\sqrt{1-m_{w}^{+} u_{a}^{+}}\right) \\
& =\frac{1}{\kappa} \ln y^{+} / y_{a}^{+}
\end{aligned}
$$

When $m_{w}^{+}=0$, Eqs. (3) with $p^{+}=0$ and (5) are reduced to the ordinary velocity distributions

$$
\begin{aligned}
u^{+} & =y^{+} \\
u^{+} & =\frac{1}{\kappa} \ln y^{+}+u_{a}^{+}-\frac{1}{\kappa} \ln y_{a}^{+} \\
& =2.5 \ln y^{+}+5.5
\end{aligned}
$$

By matching the inner edge of the turbulent region with the outer edge of the viscous sublayer at $y_{a}^{+}, u_{a}^{+}$ is given as

$$
u_{a}^{+}=-\left\{\exp \left(-m_{w}^{+} y_{a}^{+}\right)-1\right\} / m_{w}^{+}
$$

In this derivation, $p^{+}$in Eq. (3) has been neglected. When $m_{w}^{+}=0$, the matching point is given by $y_{a}^{+}=$ $u_{a}^{+}=11.7$. When $m_{w}^{+} \neq 0$, there arises a question as to how to determine the matching point. In this study, three criteria for the matching point have been examined; $y_{a}^{+}=11.7, u_{a}^{+}=11.7$ and $y_{a}^{+} u_{a}^{+}=(11.7)^{2}$. From comparison with the measured velocity distributions ${ }^{4}$, it is found that the criterion of $u_{a}^{+}=11.7$ with $\kappa=0.4$ gives better agreement with the measurements than do the other criteria. As shown in Fig. 1, agreement is excellent for the injection flow but it becomes poorer with increasing suction rate.

Equation (5) is transformed as

$$
u^{+}=\frac{1}{\kappa} \ln y^{+}+C-\frac{m_{w}^{+}}{4}\left(\frac{1}{\kappa} \ln y^{+}+C\right)^{2}
$$

where

$$
\begin{aligned}
C & =\frac{-2}{m_{w}^{+}}\left(\sqrt{1-m_{w}^{+} u_{a}^{+}}-1\right)-\frac{1}{\kappa} \ln y_{a}^{+} \\
u_{a}^{+} & =11.7 \\
y_{a}^{+} & =-\left[\ln \left(1-11.7 m_{w}^{+}\right)\right] / m_{w}^{+}
\end{aligned}
$$

Equation (9) is also compared with the measured velocity distributions in Fig. 2.

In Stevenson's expression ${ }^{13)}, C$ has been taken as independent of injection or suction rate. $\mathrm{Na}^{9}$ assumed that this is also applicable to porous tube flow. His assumption is, however, invalid at large injection or suction rate as shown by dotted lines in Fig. 2. This is due to the fact that Na's prediction overestimates the effect of wall mass flux on the friction factor, as will be described in subsection 4.2.

\section{Friction Factor}

The Reynolds number is given by

$$
R e=4 R^{+} \int_{0}^{1}(1-y / R) u^{+} d(y / R)
$$

and the friction factor is given by

$$
C_{f}=\frac{1}{2}\left[\int_{0}^{1}(1-y / R) u^{+} d(y / R)\right]^{-2}
$$

Substitution of Eqs. (3) with $p^{+}=0$ and (9) into Eq. (12) leads to the integration

$$
\int_{0}^{1}(1-y / R) u^{+} d(y / R)=I_{l}-I_{t}\left(y_{a}^{+}\right)+I_{t}\left(R^{+}\right)
$$

where $I_{l}$ denotes a definite integration in the viscous sublayer

$$
\begin{aligned}
I_{l} & =\left\{\exp \delta-1-\delta+\left[(1-\delta) \exp \delta-1+\frac{1}{2} \delta^{2}\right] / \Delta\right\} / m_{w}^{+2} R^{+} \\
\delta & \equiv-m_{w}^{+} y_{a}^{+}, \quad \Delta \equiv-m_{w}^{+} R^{+}
\end{aligned}
$$

and $I_{t}$ denotes an indefinite integration in the turbulent region

$$
\begin{aligned}
I_{t}= & \left(\frac{y}{R}\right)\left[z-\frac{1}{\kappa}-\frac{m_{w}^{+}}{4}\left(z^{2}-\frac{2}{\kappa} z+\frac{2}{\kappa^{2}}\right)\right] \\
& -\left(\frac{y}{R}\right)^{2}\left[\frac{z}{2}-\frac{1}{4 \kappa}-\frac{m_{w}^{+}}{4}\left(\frac{z^{2}}{2}-\frac{1}{2 \kappa} z+\frac{1}{4 \kappa^{2}}\right)\right]
\end{aligned}
$$

$z \equiv \frac{1}{\kappa} \ln y^{+}+C$

Using Eqs. (10), (12), (14) and (15), one can calcu- 


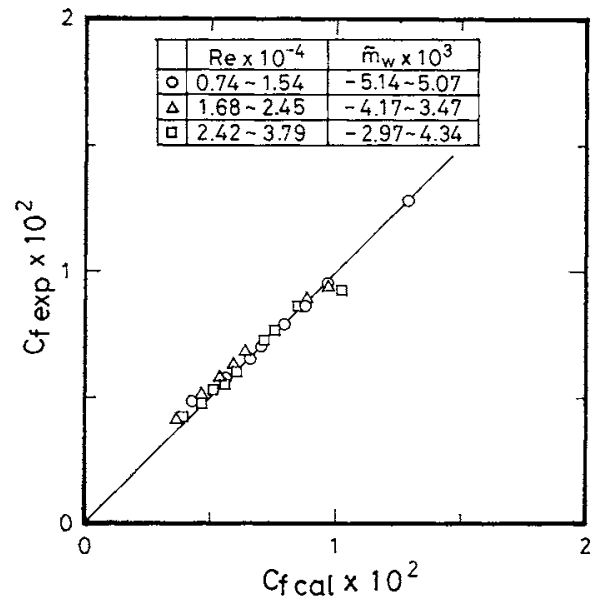

Fig. 3 Comparison of calculated $C_{f}$ with experimental results

late the value of $C_{f}$ for given $R e$ and $\tilde{m}_{w}\left(\equiv m_{w} / \rho \bar{u}\right)$. When $\tilde{m}_{w}=0$, the calculated values of $C_{f 0}$ are in good agreement with the empirical correlation by Drew, Koo and McAdams ${ }^{3}$ within 3 percent over a range of $5 \times 10^{3} \leq R e \leq 10^{8}$. The experimental results ${ }^{4)}$ of $C_{f}$ for injection or suction flow are in good agreement with the predictions, as shown in Fig. 3.

\section{Pressure Gradient}

When the self-similarity, $u(x, r) / \bar{u}(x)=\tilde{u}(r)$, is applicable to porous tube flow, integration of the equation of motion is expressed as $^{4}$

$$
\frac{d \tilde{p}}{d \tilde{x}}=-2 C_{f}+8 \tilde{m}_{w} \beta
$$

where $\beta$ is an axial momentum flux given by

$$
\beta=2 \int_{0}^{1}(1-y / R) \tilde{u}^{2} d(y / R)
$$

Substitution of Eqs. (3) and (9) into Eq. (17) gives

$$
\beta=I_{l \beta}-I_{t \beta}\left(y_{a}^{+}\right)+I_{t \beta}\left(R^{+}\right)
$$

where $I_{l \beta}$ is a definite integration in the viscous sublayer

$$
\begin{aligned}
I_{l \beta}= & \frac{C_{f}}{m_{w}^{+2}}\left[\frac{1}{\Delta}\left(\frac{1}{2} \exp 2 \delta-2 \exp \delta+\frac{3}{2}+\delta\right)\right. \\
& -\frac{1}{\Delta^{2}}\left(\frac{\Delta}{2} \exp 2 \delta-2 \delta \exp \delta-\frac{1}{4} \exp 2 \delta\right. \\
& \left.\left.+2 \exp \delta-\frac{7}{4}+-\frac{1}{2} \delta^{2}\right)\right]
\end{aligned}
$$

and $I_{t \beta}$ is an indefinite integration in the turbulent region

$$
\begin{aligned}
I_{t \beta}=C_{f} & \kappa\left(\frac{y}{R}\right)\left[F_{1}(z, \kappa)-\frac{m_{w}^{+}}{2} \cdot F_{2}(z, \kappa)+\frac{m_{w}^{+2}}{16} F_{3}(z, \kappa)\right] \\
& -\left(\frac{y}{R}\right)^{2}\left[F_{1}(z, 2 \kappa)-\frac{m_{w}^{+}}{2} F_{2}(z, 2 \kappa)\right. \\
& \left.\left.+\frac{m_{w}^{+2}}{16} F_{3}(z, 2 \kappa)\right]\right\} \\
F_{1}(z, \kappa) & \equiv z^{2} / \kappa-2 z / \kappa^{2}+2 / \kappa^{3}
\end{aligned}
$$

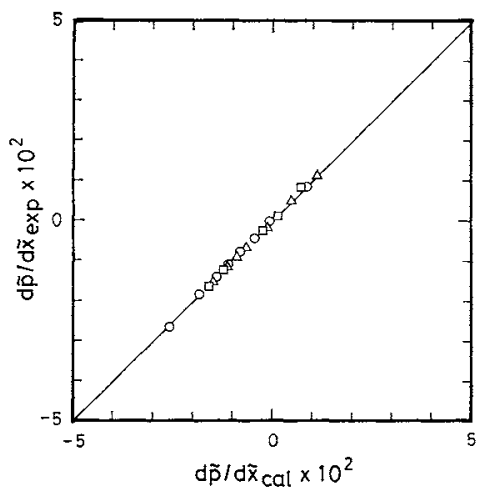

(Keys are the same as those in Fig. 3.)

Fig. 4 Comparison of calculated $d \tilde{p} / d \tilde{x}$ with experimental results

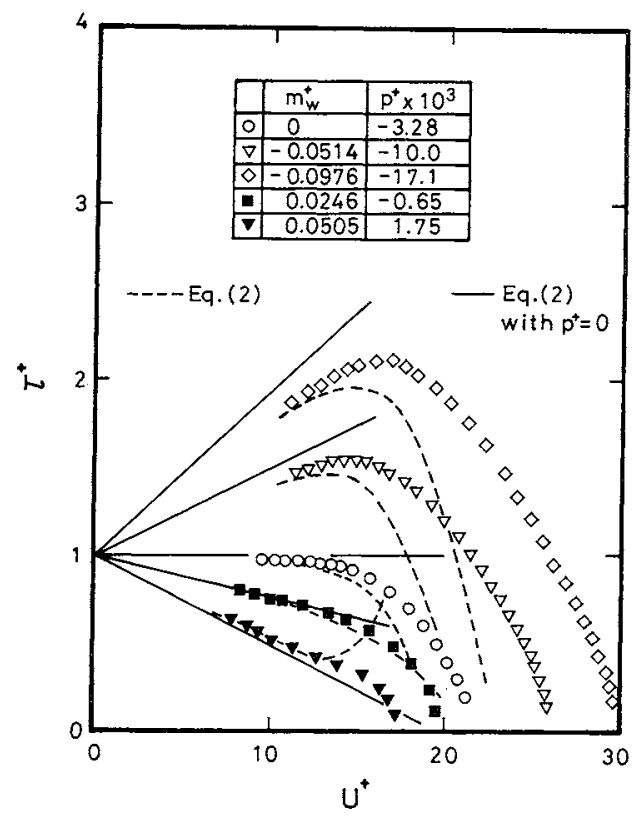

Fig. 5 Comparison of Eq. (2) with experimental results of shear stress

$$
\begin{aligned}
& F_{2}(z, \kappa) \equiv z^{3} / \kappa-3 z^{2} / \kappa^{2}+6 z / \kappa^{3}-6 / \kappa^{4} \\
& F_{3}(z, \kappa) \equiv z^{4} / \kappa-4 z^{3} / \kappa^{2}+12 z^{2} / \kappa^{3}-24 z / \kappa^{4}+24 / \kappa^{5}
\end{aligned}
$$

One can estimate the value of $d \tilde{p} / d \tilde{x}$ from these equations. The experimental results ${ }^{4}$ are in good agreement with the predictions, as shown in Fig. 4.

\section{Discussion}

\section{1 Examination of the assumptions}

The variation of axial momentum has been neglected in Eq. (2) and the mixing length relation $l=0.4 y$ has been adopted in deriving Eq. (5) from Eq. (2) with $p^{+}=0$. In the following, applicabilities of Eq. (2) and the mixing length relation will be discussed.

Equation (2) is compared with the experimental results ${ }^{4}$ by the dotted curves in Fig. 5. Except for $m_{w}^{+}=0.0246$, its applicability is restricted to a region very 


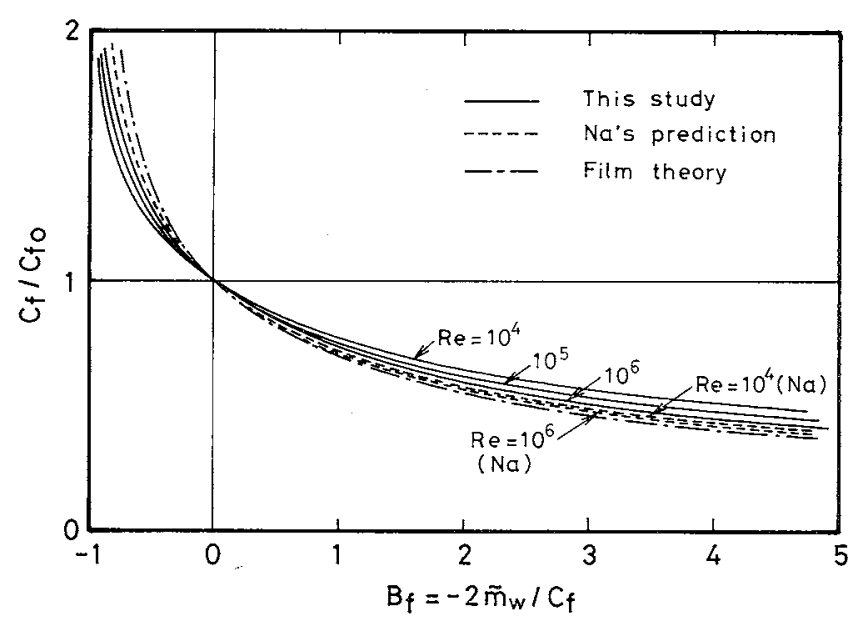

Fig. 6 Variation of friction factor against wall mass flux

near the wall. Equation (2) with $p^{+}=0$ expressed by the solid line goes on increasing from the wall shear stress in injection flow and decreasing in suction flow. Since $p^{+}$decreases with increasing injection rate, as will be shown in Fig. 7 , Eq. (2) with $p^{+}=0$ gives larger values for injection flow, especially in the turbulent region far from the wall. In suction flow, $p^{+}$becomes very small and then increases with suction rate, so Eq. (2) with $p^{+}=0$ gives smaller values with increasing suction rate.

On the other hand, the mixing length relation $l=$ $0.4 y$ tends to give larger values than the measured ones with increasing distance from the wall, as shown in Fig. 10 in the previous paper ${ }^{4}$.

In spite of these facts that both of Eq. (2) and the mixing length relation are not good approximations in the turbulent region, Eq. (5) agrees well with the measurements, especially for injection flow. It may be that the simultaneous adoption of Eq. (2) with $p^{+}=0$ and $l=0.4 y$ has the effect of canceling the above-mentioned disagreement of local shear stress and mixing length together with the criterion of $u_{a}^{+}=$ 11.7 for the matching point. But this canceling effect becomes inactive at $m_{w}^{+}=0.0505$, resulting in the poor agreement of Eq. (5) or (9) with measured velocity distribution in Fig. 1 or 2.

Under the condition of high suction rate, the shear stress given by Eq. (2) with $p^{+}=0$ might become zero at some radial position other than the tube center. This suggests that there is a limiting condition in applying Eq. (5) or (9) to suction flow, in which the velocity distribution becomes flatter with increasing suction rate. A limiting flow is such that the shear stress is zero at $y_{a}^{+}$and the velocity distribution is flat and almost equal to the average velocity $\vec{u}$ at $y^{+} \geq y_{a}^{+}$. From Eq. (2) with $p^{+}=0, \quad m_{w}^{+}=1 / u_{a}^{+}=$ $\sqrt{C_{f} / 2}$ or $\tilde{m}_{w}=C_{f} / 2=1 / u_{\alpha}^{+2}=7.3 \times 10^{-3}$ is obtained as the limiting condition, and the applicability of Eq. (5) or (9) is restricted to a condition of $\tilde{m}_{w}<7.3 \times 10^{-3}$.

\section{2 Friction factor}

With increasing Reynolds number, $I_{l}$ and $I_{t}\left(y_{a}^{+}\right)$in Eq. (13) become much smaller than $I_{t}\left(R^{+}\right)$and then $C_{f}$ is simply expressed by

$$
C_{f}=\frac{1}{2} I_{t}\left(R^{+}\right)^{-2}
$$

which is transformed into Na's expression ${ }^{9}$ as

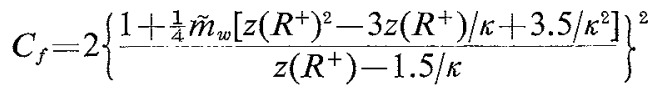

At $\tilde{m}_{w}=0$, Eq. (22) is reduced to Prandtl's universal law of friction ${ }^{11}$. At $R e=10^{5}, C_{f}$ calculated by Eq. (22) agrees with $C_{f}$ by the full equation, Eq. (13), within 1 percent in a range of $-1 \times 10^{-2}<\tilde{m}_{w}<7 \times$ $10^{-3}$. The deviation increases with decreasing Reynolds number and with increasing suction rate, and Eq. (22) gives an about 10 percent lower value to $C_{f}$ at $R e=5 \times 10^{3}$ and $\tilde{m}_{w}=7 \times 10^{-3}$. But this deviation is reduced to 4 percent when $C_{f 0}$ is also calculated by Eq. (22) and $C_{f}$ is compared in the form of $C_{f} / C_{f 0}$. It may be concluded that Eq. (22) is valid in predicting the effect of wall flux on $C_{f} / C_{f 0}$.

In Fig. 6, $C_{f}$ is compared in the form of $C_{f} / C_{f 0}$ vs. $B_{f}\left(\equiv-2 \tilde{m}_{w} / C_{f}\right)$. The prediction in this study indicates that the effect of wall mass flux becomes more remarkable with increasing Reynolds number. $\mathrm{Na}$ 's prediction with $C=5.5$ and the film theory ${ }^{2}$ overestimate the effect of wall mass flux.

\section{3 Pressure distribution}

At large Reynolds number, $\beta$ can be approximated by $I_{t \beta}\left(R^{+}\right)$or Na's expression ${ }^{9}$.

$$
\begin{aligned}
\beta= & C_{f}\left[\frac{z\left(R^{+}\right)^{2}}{2}-\frac{3 z\left(R^{+}\right)}{2 \kappa}+\frac{7}{4 \kappa^{2}}\right. \\
& -\frac{m_{w}^{+}}{2}\left(\frac{z\left(R^{+}\right)^{3}}{2}-\frac{9 z\left(R^{+}\right)^{2}}{4 \kappa}+\frac{21 z\left(R^{+}\right)}{4 \kappa^{2}}-\frac{45}{8 \kappa^{3}}\right) \\
& +\frac{m_{w}^{+2}}{16}\left(\frac{z\left(R^{+}\right)^{4}}{2}-\frac{3 z\left(R^{+}\right)^{3}}{\kappa}+\frac{21}{2} \frac{z\left(R^{+}\right)^{2}}{\kappa^{2}}\right. \\
& \left.\left.-\frac{45 z\left(R^{+}\right)}{2 \kappa^{3}}+\frac{93}{4 \kappa^{4}}\right)\right]
\end{aligned}
$$

The pressure gradient $d \tilde{p} / d \tilde{x}$ calculated by Eqs. (16), (22) and (23) agrees with that by the full equation within 1 percent at $-1 \times 10^{-2} \leq \tilde{m}_{w} \leq 7 \times 10^{-3}$ and $5 \times$ $10^{3} \leq \operatorname{Re} \leq 10^{7}$.

Mizushina et al $^{81}$ presented an empirical correlation with $d \tilde{p} / d \tilde{x}$ by neglecting the dependency of the Reynolds number as

$$
d \tilde{p} / d \tilde{x}=-0.0115+5.731 \tilde{m}_{w}-103.5 \tilde{m}_{w}^{2}+3420 \tilde{m}_{w}^{3}
$$

As shown in Fig. 7, the prediction of $d \tilde{p} / d \tilde{x}$ has been improved and $d \tilde{p} / d \tilde{x}$ increases with increasing Reynolds number.

\section{Conclusion}

The velocity distribution in the turbulent region of the fully developed flow is well expressed by Eq. (5) or (9), especially for injection flow. The coefficient 
$C$ in Eq. (9) is not constant but is determined by Eq. (10).

The analytical expressions derived in sections 2 and 3 are applicable to the prediction of $C_{f}$ and $d \tilde{p} / d \tilde{x}$, and they are reduced to Eqs. (22) and (23) at $R e>10^{5}$.

Applicability of the expressions derived in this analysis is restricted to a flow at $\tilde{m}_{w}<7.3 \times 10^{-3}$.

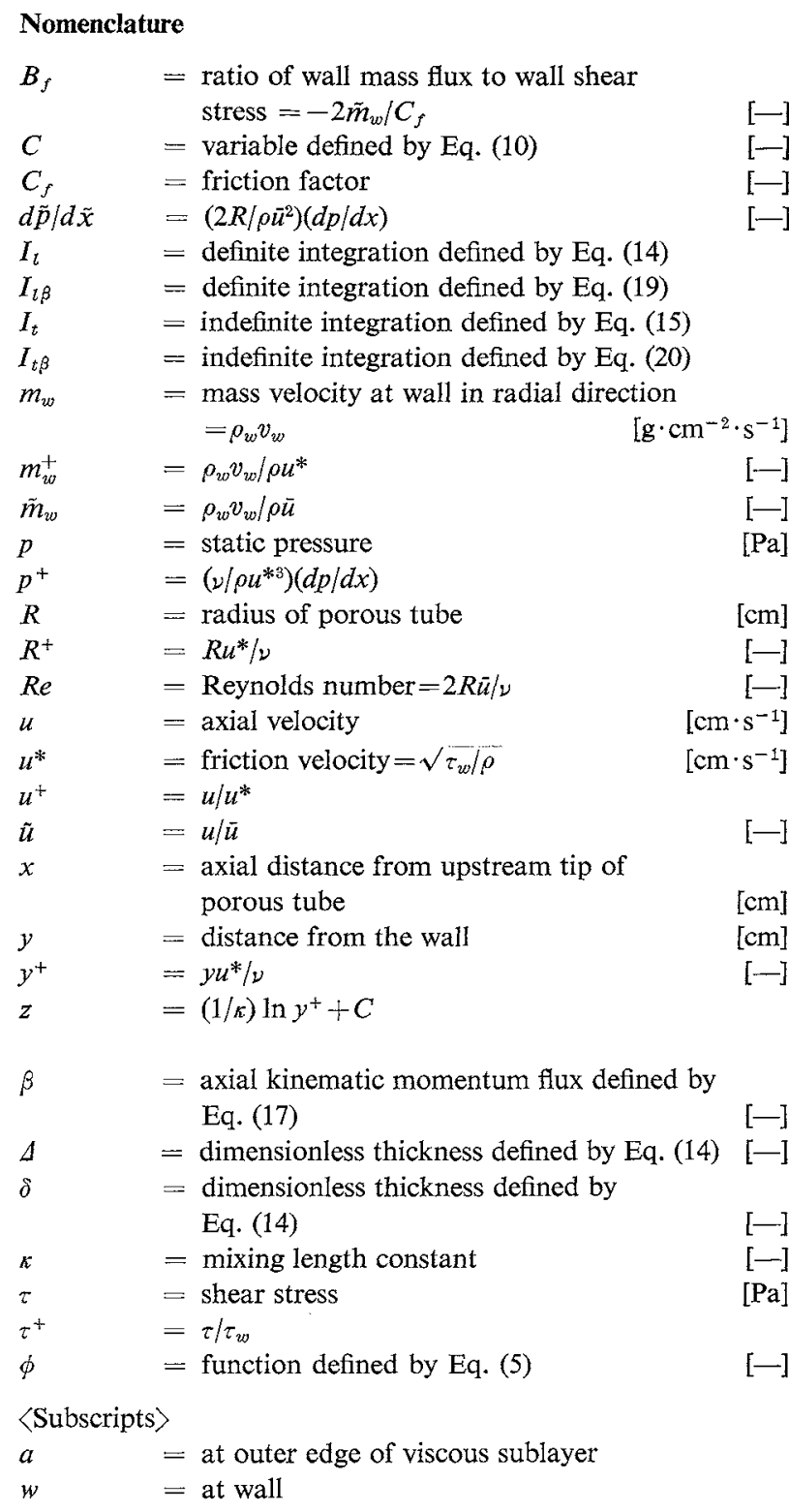

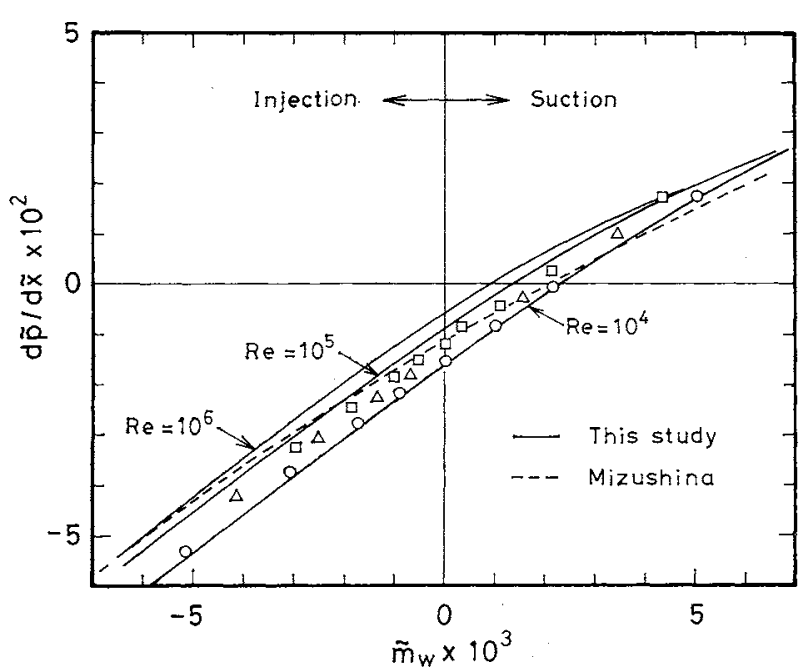

(Keys are the same as those in Fig. 3.)

Fig. 7 Dependency of $d \tilde{p} / d \tilde{x}$ on $\operatorname{Re}$ and $\tilde{m}_{w}$

$0 \quad=$ without injection or suction

\section{Literature Cited}

1) Aggarwal, J. K., M. A. Hollingsworth and Y. R. Maythew: Int. J. Heat Mass Transfer, 15, 1585 (1972).

2) Bird, R. B., W. E. Stewart and E. N. Lightfoot: "Transport Phenomena", p. 658, John Wiley \& Sons, Inc. (1960).

3) Drew, T. B., E. C. Koo and W. H. MacAdams: $A I C h E$, 28, 56 (1932).

4) Ito, R., Y. Hirata, J. Ikeda, N. Imamura and K. Sakata: J. Chem. Eng. Japan, 12, 91 (1979).

5) Kinny, R. B. and E. M. Sparrow: Trans. ASME, Ser. C, 92, 117 (1970).

6) Merkine, L., A. Solan and Y. Winograd: ibid., 93, 242 (1971).

7) Mizushina, T., S. Takeshita and G. Unno: J. Chem. Eng. Japan, 4, 135 (1971).

8) Mizushina, T., F. Ogino and S. Takeshita: ibid., 8, 217 (1975).

9) Na, T. Y.: Trans. ASME, Ser. D, 700 (1972).

10) Rubesin, M. W.: NACA TN 3341 (1954).

11) Schlichting, H.: "Boundary Layer Theory", 7th ed. p. 609, McGraw-Hill (1979).

12) Simpson, R. L.: J. Fluid Mech., 42, part 4, 769 (1970).

13) Stevenson T. N.: AIAA J, 6, 553 (1968).

14) Torii, K., N. Nishiwaki and M. Hirata: Proc. of 3rd International Heat Transfer Conf., Vol, III, 34 (1966).

15) Weissberg, H. L. and A. S. Berman: Proceedings of the Heat Transfer and Fluid Mechanics, 14, 1 (1955). 